\title{
Bioavailable Lead in Topsoil Collected from General Aviation Airports
}

\author{
Jerry H. Kavouras, Randal J. DeMik, and Allison C. Hopkins \\ Lewis University
}

\begin{abstract}
Representatives of the aviation community are interested in determining the environmental effects of leaded fuel emissions from general aviation flight operations. Soil is an environmental sink for lead to accumulate over time. This study measured bioavailable lead concentrations in topsoil at three general aviation airports and at one site with no general aviation operations in order to determine if general aviation aircraft operations are contributing to the contamination of airport topsoil at levels that require remediation by law. Bioavailable refers to substances that organisms can absorb, because they have access to them. Topsoil was collected from refueling areas, run-up areas, and approach corridor/departure end runways. The samples were dried and the fine fractions were separated. The fine fractions were suspended in nitric acid to extract the lead for laboratory analysis. A univariate ANOVA was computed comparing the quantity of soluble lead in the topsoil from three general aviation airports (further classified as high, moderate, and low volume of flight operations) and at one site with no general aviation operations (arboretum). A significant main effect for the high volume airport was found $(F[2,24]=10.966, p<.05)$, in which the lead in topsoil samples from high volume airports were lower than the samples from the other airports, but similar to the arboretum. Another analysis was calculated comparing quantity of soluble lead in topsoil at sites within airports (refueling areas, run-up areas, and approach corridor/departure end runways). No significant main effect was found among the sites $(F[4,24]=1.065, p>$ $.05)$. However, an interaction was observed when comparisons were made between the airport sites $(n=3)$ and locations sampled at the sites $(n=3)$ that was significant $(F[4$, $24]=2.902, p<.05)$. Tukey's HSD was used to determine the nature of the differences. The analysis revealed that concentrations of lead in soil samples collected from the high volume airport refueling area, run-up area, and approach corridor/departure end runway were significantly lower than the moderate volume airport run-up area lead concentrations. The results at the three airports investigated support the conclusions that the risk of ingesting lead from surface soils is low and bioavailable lead in the soil sampled from general aviation airports is not accumulating in quantities that require remediation according to Environmental Protection Agency requirements.
\end{abstract}

\section{Introduction}

Representatives of the aviation community are interested in determining the environmental effects of leaded fuel emissions from general aviation flight operations because lead is a neurotoxin and there are potential consequences to public health. The Institute of Medicine (1998) defined public health as, "what we as a society do collectively to assure conditions in which people can be healthy" (p. 19). The majority of general aviation piston-engine powered aircraft use aviation gasoline (Avgas) that is designated as 100 Low Lead (100LL). According to Schaufele (2008), more than 
200,000 general aviation aircraft burn approximately 190 million gallons of 100LL annually. This exhaust makes up $45 \%$ of the total lead emissions in the air over the United States.

Early automotive engineers discovered that gasoline has a tendency to "knock" that is, explode rapidly instead of burning smoothly inside engine cylinders. According to Midgley (1937), knocking was eliminated when the chemical compound tetra-ethyl lead (TEL) was added to gasoline because it reduces temperature and pressure build up within cylinders. Lead additives in gasoline prevent detonation, which causes a high-pressure spike in the combustion chamber (Cline, 2000) and eventually engine damage (Harvey, 2006). The discovery of leaded fuel allowed for the development of powerful high compression engines. As leaded fuel was found to be very effective in high compression engines, its use spread from the automotive to the aviation industry.

When TEL was first added to fuel, there was no information about the negative health consequences of lead exposure. Recognition of TEL as a toxic substance has guided the phase-out of leaded gasoline in automobiles that began in 1970 in the United States (Matsui, 2007). According to the Agency for Toxic Substances \& Disease Registry (2007), lead can affect every organ in the human body and exposure to high levels of lead can damage the brain, kidneys, and nervous system. A 2001 report by the Environmental Protection Agency (EPA) defined a soil lead hazard as "bare soil that contains $400 \mathrm{ppm}$ of lead in a play area or $1200 \mathrm{ppm}$ in other parts of a yard" (p. 21). Less than $400 \mathrm{ppm}$ is considered urban background and does not require remediation (EPA, 2001). Young people are more susceptible to lead poisoning, and even small amounts of lead ingestion can impair their mental and physical growth (U.S. Department of Health and Human Services, 2007).

Lead is not only harmful to humans, but wildlife as well. Predators accumulate higher pollutant concentrations than their contaminated prey because of bio-magnification. As a result, the EPA (2011) determined that "people and other animals at the top of the food chain who eat contaminated fish or meat are exposed to concentrations that are much higher than the concentrations in the water, air, or soil" (p. 13).

Soil is an environmental sink for lead to accumulate over time. Anthropogenic sources of lead deposited in soil typically remain at high levels because they do not biodegrade or decay, and plants do not absorb them quickly (EPA, 2001). According to Howden, Schneider, and Grosser (1996), lead paints used on deteriorating exterior surfaces for homes built before 1980 can highly contaminate soils. Lead concentrations in soils near roadways exposed to leaded gasoline over time can be 30 to 2000 times greater than natural levels, particularly older roadways within large cities (Howden et al., 1996).

Piston-engine-equipped general aviation aircraft still use leaded fuel. The EPA estimates that between 1970 and 2007, the combustion of avgas in general aviation has released 34,000 tons of lead into the atmosphere and affects three million children who attend school near aviation facilities (EPA, 2010). The EPA has issued an advance notice of proposed rulemaking (ANPR) addressing engine emission standards for piston aircraft 
(EPA, 2010). Awareness of the negative health and environmental consequences of leaded fuel and the recent ANPR are prompting research towards developing an unleaded alternative to avgas.

\section{Statement of the Problem}

Aviation gasoline or avgas achieves higher octane ratings by the addition of tetra-ethyl lead (TEL), a toxic substance that was phased out for automobile use in the United States. Few studies have provided empirical evidence of lead contamination in soil from general aviation operations that continue to use avgas containing TEL. This study will measure soluble (bioavailable) lead concentrations in topsoil at three general aviation airports and at one non-airport site located several miles from any general aviation operations in order to determine if general aviation aircraft operations are contaminating the airport topsoil at levels that require remediation by law. Use of the term "bioavailable" in this study refers to substances that organisms can absorb, because they have access to them. The methods in this study allowed the investigators to measure lead that is soluble using nitric acid extraction (Howden et al., 1996). Soluble lead is potentially available to soil organisms for ingestion or uptake. This study tested the following research hypotheses:

1. Airports with higher volumes of general aviation operations would have greater quantities of soluble lead in the topsoil compared to airports with lower volumes of general aviation operations.

2. Sites (refueling area, run-up area, and runway approach corridor/departure end) within each general aviation airport differ in the quantity of soluble lead in the topsoil.

3. The quantities of soluble lead in the topsoil of general aviation airports are greater than or equal to levels that require remediation as established by the EPA.

\section{Methodology}

\section{Sites}

The sampling sites are three general aviation airports in northeastern Illinois that are distinguished based on numbers of annual departures and arrivals. The Airport Inventory Report (Illinois Department of Transportation, 2012) listed annual operations for Airport 1 as 104,000, Airport 2 as 50,000, and Airport 3 as 25,000. The Airport Inventory Report refers to operations as the number of take-offs and landings at an airport. The majority of operations at the selected airports were piston engine aircraft. The researchers classified the airports as high (Airport 1), moderate (Airport 2), and low (Airport 3) volume based on the data. Soil samples were collected near the highest use approach corridor/departure end runway, run-up areas, and refueling areas (see Figures 1-3). The approach corridor is the same as the take-off point, where emissions from aircraft exhaust are the greatest discharge due to high power settings at beginning of take-off roll. At airports with multiple runways, the run-up area and approach corridor/departure end were sampled at the runway of primary use. The nearest topsoil to paved areas was collected in this study. Samples were also collected from forested areas within the Morton Arboretum as a 
control since there is no direct contact with airplanes. The sites within the arboretum were Location $1\left(41^{\circ} 49^{\circ} 07^{\circ} \mathrm{N}, 88^{\circ} 05^{\circ} 02 \mathrm{~W}\right)$, Location $2\left(41^{\circ} 48^{\circ} 52^{\circ} \mathrm{N}, 88^{\circ} 02^{\circ} 34^{\circ} \mathrm{W}\right)$, and Location $3\left(41^{\circ} 49^{\circ} 09^{\circ} \mathrm{N}, 88^{\circ} 03^{\circ} 21^{\circ} \mathrm{W}\right)$.

\section{Procedures}

Each site was visited during a normal operational day (meaning no special events, air shows, or fly-ins, which would have a direct effect of the number of planes). A coring sampler (LaMotte Company) was bored into the ground at a depth of 1.5 to 2 inches. The top $1 / 2$ inch of soil was placed into a sterile Whirlpak bag (Howden et al., 1996). Each sample consisted of three samplings within a one-foot diameter of the initial sampling that were placed into the same Whirlpak bag (Fig. 4). To reduce contamination, excess soil was wiped from the sampling tube between sampling using a gloved finger and then the tube was washed with distilled water and wiped with clean paper towels. Three samples were collected from each location (Fig. 4). The samples were transported to the Biology Department at Lewis University and stored at room temperature. According to Materials Analytical Services Inc. (n.d.), no refrigeration or preservation was required.

\section{Measures}

Soil samples were dried using a conventional microwave. Dried soil samples were separated into fine and coarse fractions using two sieves - a $4.75 \mathrm{~mm}$ (No. 4) sieve to remove large debris, e.g. rocks and sticks, and a $250 \mu \mathrm{m}$ (No. 60) sieve to separate coarse and fine fractions of soil. The fractions were weighed, placed into sterile Whirlpak bags, and stored at room temperature until ready for use.

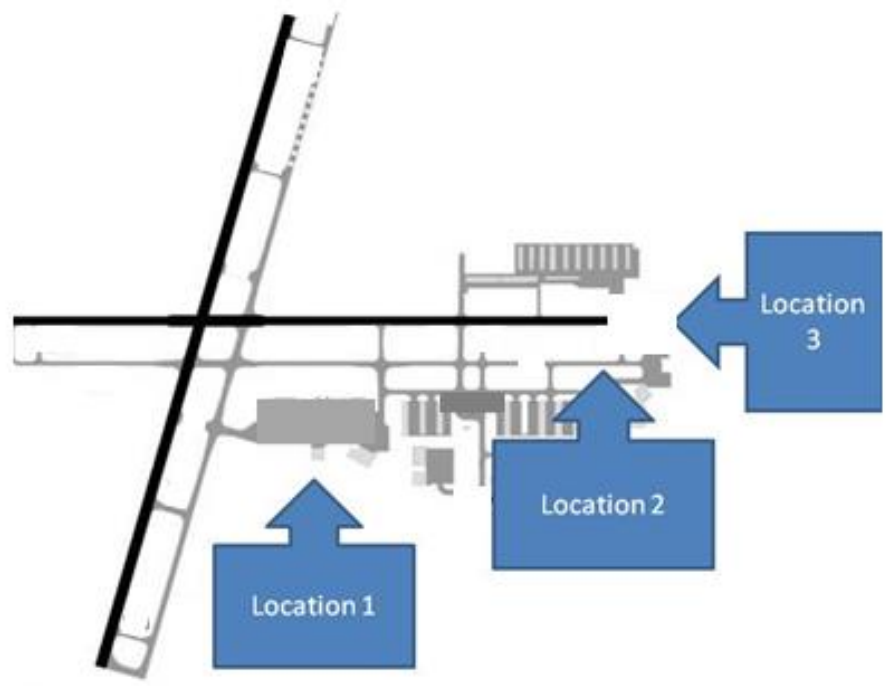

Figure 1. Map of high volume airport sample site locations. Soil samples were collected from the refueling area (Location 1), the run-up area (Location 2), and the approach corridor/departure end runway (Location 3). 


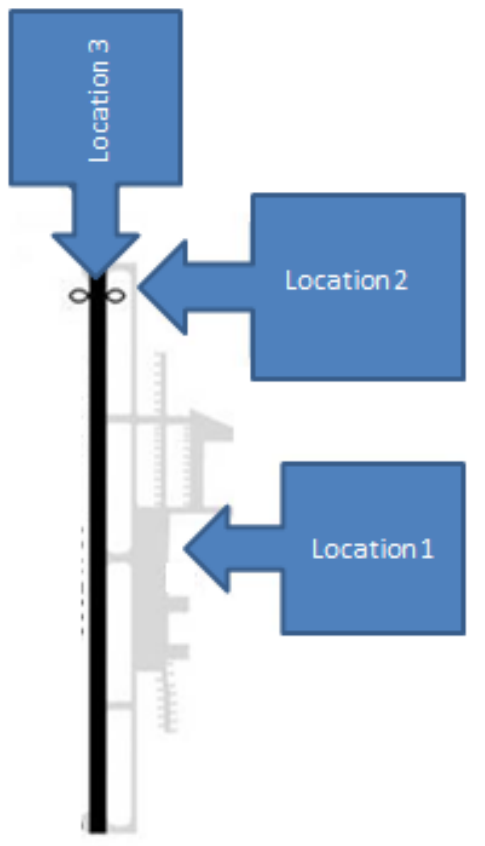

Figure 2. Map of moderate volume airport sample site locations. Soil samples were collected from the refueling area (Location 1), the run-up area (Location 2), and the approach corridor/departure end runway (Location 3).

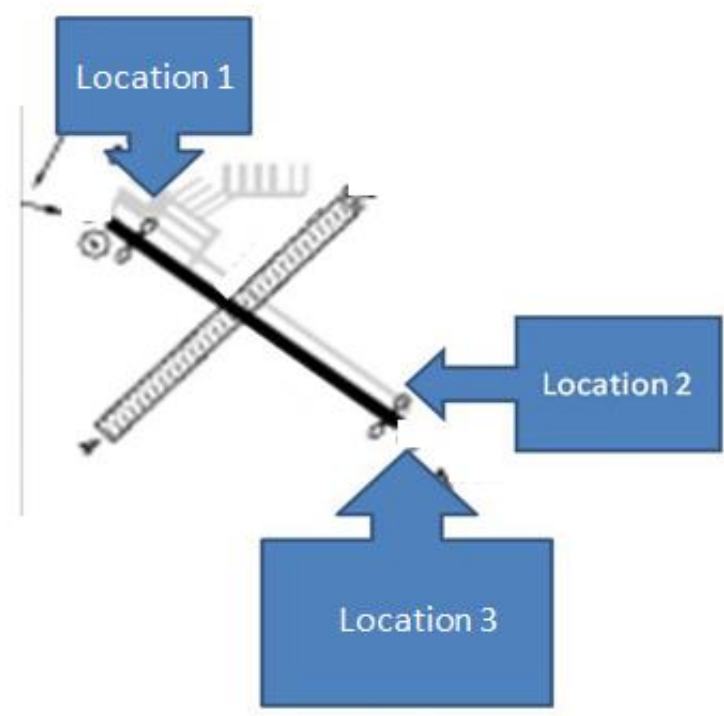

Figure 3. Map of low volume airport sample site locations. Soil samples were collected from the refueling area (Location 1), the run-up area (Location 2), and the approach corridor/departure end runway (Location 3). 


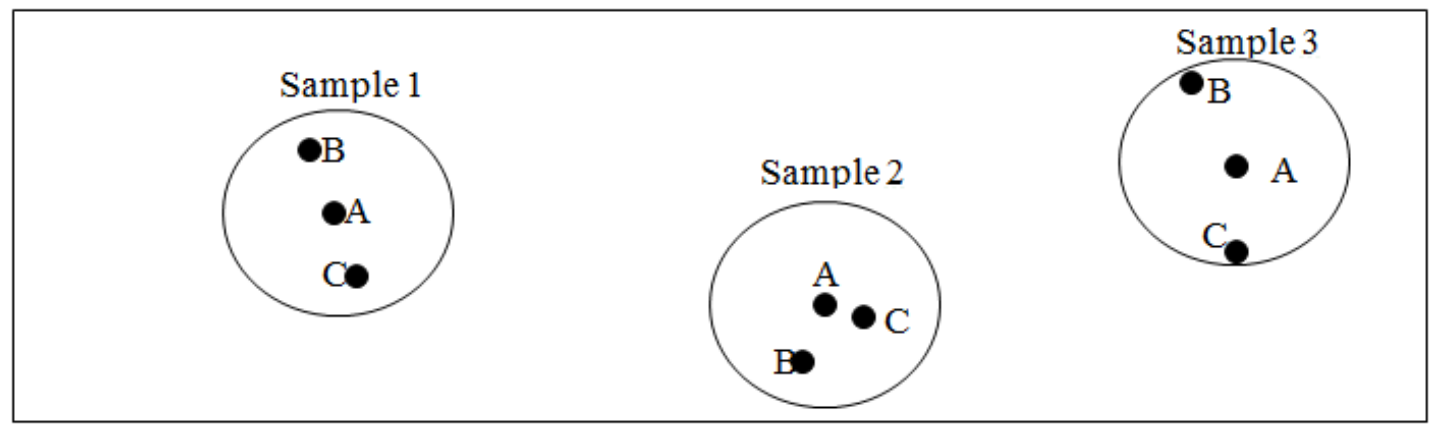

Figure 4. An example of three samples taken from one location within a site. The large circle is one foot in diameter from the initial sampling (A), whereas the other samples (B, C) are taken within the circle. The large circle represents one sample.

The investigators were able to measure the lead concentration in fine fractions using nitric acid extraction (Howden et al., 1996). Generally, fine fractions are the source of ingested lead because particles of this size sticks to hands and objects, such as clothing, which transfer lead to the mouth (EPA, 2000). Three grams of the fine fraction were added to $30 \mathrm{~mL}$ of $1 \mathrm{M}$ nitric acid. The mixture was shaken for one hour on an orbital shaker to extract the lead from the soil. Then the mixture was centrifuged $(1000 \mathrm{x} \mathrm{g}, 10$ minutes) and the aqueous supernatant was removed for analysis. The lead concentration was determined for each fine fraction using the Pocket Colorimeter II Test Kit (Hach Company) according to the manufacturer's instructions. Lead nitrate was used as a positive control and to calibrate the colorimeter.

\section{Statistical Analysis}

The data were analyzed using a multi-factorial analysis of variance. The independent variables were the sites (High, Moderate, and Low Volume Airports, and Morton Arboretum) and the locations within the sites (refueling area, run-up area, and approach corridor/departure end). The dependent variable was the bioavailable lead concentration in the soil measured in parts per million (ppm). IBM SPSS Statistic 19 software analyzed the data. A $p$ value $<0.05$ was chosen to determine significance.

\section{Results}

The first hypothesis sought to determine if the volume of arrivals and departures at a general aviation airport relate to the quantity of soluble lead in the topsoil. A univariate ANOVA was computed comparing the quantity of soluble lead in the topsoil from three general aviation airports (further classified as high, moderate, and low volume of flight operations) and at one site with no general aviation operations (arboretum). A significant main effect for the high volume airport was found $(F(2,24)=10.966, p<$ $.05)$, in which the lead in topsoil samples from high volume airports were lower than the samples from the other airports, but similar to the arboretum (refer to Table 1 and Figure $5)$. The concentrations of lead in samples collected from the high volume airport $(m=$ $0.286, s d=0.147)$ were lower than the samples from the low $(m=0.677, s d=0.264)$ and 
moderate $(m=0.763, s d=0.39)$ volume airports but similar to concentrations from the arboretum $(m=0.498, s d=0.147)$.

The second hypothesis sought to determine if sites within each general aviation airport vary in the quantity of soluble lead in topsoil. Another analysis was calculated comparing quantity of soluble lead in topsoil at sites within airports (refueling areas, run-up areas, and approach corridor/departure end runways). No significant main effect was found among the sites $(F(4,24)=1.065, p>.05$, refer to Table 1$)$. However, an interaction was observed when comparisons were made between the airport sites $(n=3)$ and locations sampled at the sites $(n=3)$ that was significant $(F(4,24)=2.902, p<.05$, refer to Table 1 and Figure 6). Tukey's HSD was used to determine the nature of the differences. This analysis revealed that concentrations of lead in soil samples collected from the high volume airport refueling area $(m=0.2577, s d=0.23897)$, run-up area $(m=0.25, s d=$ $0.14)$, and approach corridor/departure end runway $(m=0.35, s d=0.025)$ were significantly lower than the moderate volume airport run-up area $(m=1.07, s d=0.506)$ lead concentrations.

The third hypothesis sought to determine if the quantity of soluble lead in the topsoil of general aviation airports meet levels that require remediation. The levels of lead in this study were considerably lower than the levels established by federal agencies (refer to Figures 5 \& 6).

Table 1

Two-factor analysis of variance of lead levels in soil samples.

\begin{tabular}{l|cccccc}
$\begin{array}{l}\text { Source } \\
\text { Variation }\end{array}$ & of & df & SS & MS & F & $\boldsymbol{P}$ \\
\hline Intercept & 1 & 106687.289 & 106687.289 & 200.159 & $<0.001$ \\
Site & 2 & 11690.081 & 5845.040 & 10.966 & $<0.001$ \\
Location & 4 & 2270.783 & 567.696 & 1.065 & 0.395 \\
Site x Location & 4 & 6186.213 & 1546.553 & 2.902 & 0.043 \\
\cline { 3 - 6 }
\end{tabular}




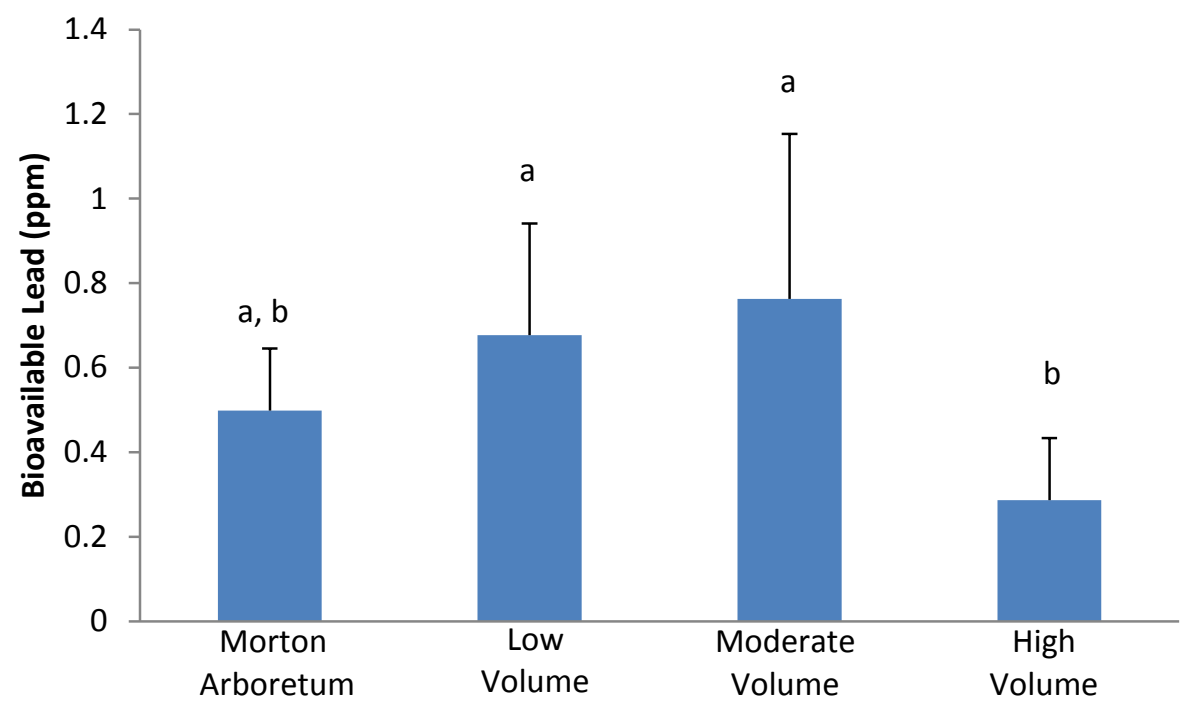

Figure 5. Lead concentrations in soil samples collected from Morton Arboretum and general aviation airports. Each bar represents the mean of all samples collected from the site. Error bars are standard deviations. The letters above the bars denote similarities among the sites in regards to lead concentrations $(p>0.05)$.

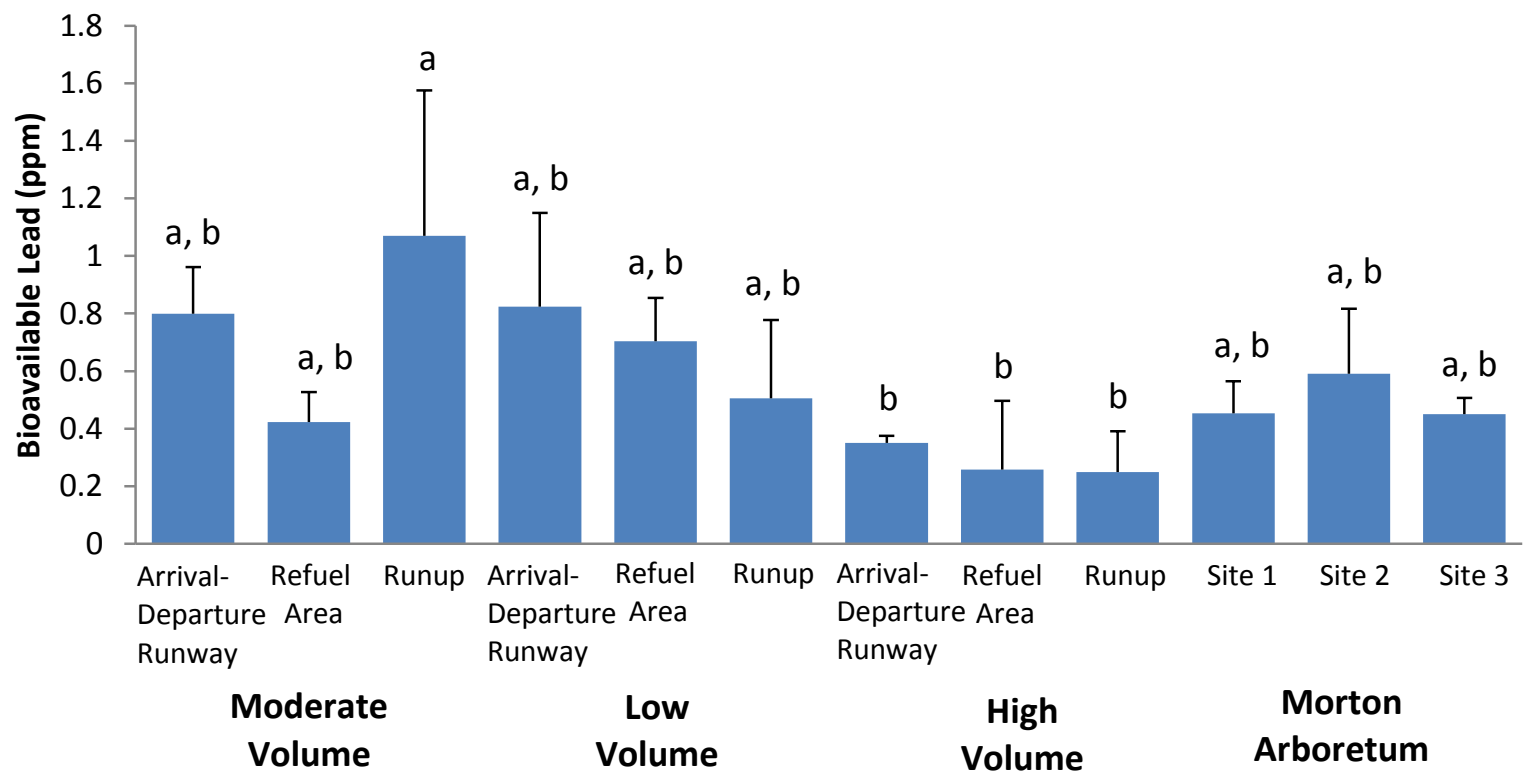

Figure 6. Lead concentrations in soil samples sorted by site and location. Each bar represents the mean of all samples collected from the location within the site. Error bars are standard deviations. The letters above the bars denote similarities among the sites in regards to lead concentrations $(p>0.05)$. 


\section{Discussion of the Results}

The overall purpose of this soil lead study was to determine if general aviation operations increase the quantity of lead in airport soils. The study examined three airports based on traffic volume and Morton Arboretum, which is not exposed directly to general aviation. The soil lead concentrations at the airports should increase with traffic volume if there was a positive correlation between general aviation operations and lead in the soil. The trend appears to be present, steadily increasing from Morton Arboretum to the moderate volume airport, until one examines the high volume airport, which is the lowest of the four sites (refer to Figure 5). Why would this trend disappear when reaching the high volume airport? First, it was discovered that the sites within the high volume airport were reconstructed recently and the topsoil was replaced. This may explain the discrepancy, because the topsoil would not have been exposed to general aviation operations using leaded fuels for an extended period. The trend suggests that traffic volume at general aviation airports increases soil lead levels, but not significantly (refer to Figure 5). Secondly, the high volume airport had multiple runways (refer to Figure 1) leading to emissions not concentrated in one area by spreading operations for engine run-up, arrivals, and departures as compared to the one runway configuration at the moderate and low volume airports where operation areas were concentrated (refer to Figures 2 \& 3). Interpretation of the data strongly suggests that both factors, reconstruction and runway configurations, are important in soil lead accumulation.

The concentrations of lead in soils were also lower than the levels defined as a soil lead hazard by the EPA (refer to Figures $5 \& 6$ ). As stated in the introduction, a soil lead hazard is bare soil that contains $400 \mathrm{ppm}$ of lead in a play area or $1200 \mathrm{ppm}$ in other parts of a yard, and remediation is not required for levels below $400 \mathrm{ppm}$. The run-up at the low volume airport had the highest soil lead concentration in this study at approximately $1.1 \mathrm{ppm}$ (refer to Figure 6). The topsoil at these sites was not bare, so the chance of ingestion was low. Some of the areas within the airports had greater levels of soil lead than other areas within the site, but they were not significantly different (refer to Figure $6)$.

The findings are consistent with those of Taylor, Park, Murphy, and Mortvedt (2010), which reported concentrations of soil lead at the perimeter of an airport in quantities well below $400 \mathrm{ppm}$. The concentrations of lead reported by Taylor et al. (2010) were greater than reported in this study; however, both were less than urban background, which supports the view that soil lead at general aviation airports does not exceed levels that require remediation. Therefore, people are not at risk of exposure to high levels of lead through ingestion of fine soil particles that could be dislodged from surfaces during routine maintenance operations.

\section{Recommendations for Future Research}

Soluble lead concentrations in fine fractions of topsoil collected from general aviation airports were below the level required for remediation by the EPA. Future work could include the coarse fractions of topsoil collected from these sites in order to create a complete soil profile. The literature recommends the fine fraction for lead analysis 
because it provides an accurate assessment of the risk from incidental ingestion; however, it does state that some soils do have higher concentrations in coarse fractions.

The three airports in northeastern Illinois that varied in traffic volume were a good start. A more complex study could be performed to confirm that traffic volume and soil lead levels are correlated, and to determine if other factors, such as length of time exposed to general aviation, runway configurations, and reconstruction are significant.

\section{Acknowledgements}

Special thanks to Tyler Dean for his assistance during sample collection and lead analysis, and Dr. Salim Diab for his technical expertise. This research was sponsored, in part, by a grant from The Colonel Stephen S. And Lyla Doherty Center for Aviation and Health Research. 


\section{References}

Agency for Toxic and Substances Disease Registry. (2007). ToxFAQs for lead (CAS \# 7439921). Retrieved from http://www.atsdr.cdc.gov/tfacts13.pdf

Cline, A. (2000). Engine Basics: Detonation and Pre-Ignition. Contact Magazine, 54 Retrieved from http://www.contactmagazine.com/Issue54/EngineBasics.html

Environmental Protection Agency. (2000). Short sheet: TRW Recommendations for sampling and analysis of soil at lead (Pb) sites. (EPA/540-F-00-010). Retrieved from http://epa.gov/superfund/lead/products/sssiev.pdf

Environmental Protection Agency. (2001). Lead-safe yards: Developing and implementing a monitoring, assessment, and outreach program for your community. (EPA/625/R-00/012). Retrieved from http://www.epa.gov/region1/leadsafe/pdf/chapter3.pdf

Environmental Protection Agency. (2010). Advance notice of proposed rulemaking on lead emissions from piston-engine aircraft using leaded aviation gasoline: Regulatory announcement (EPA420-F-10-013). Retrieved from http://www.epa.gov/nonroad/aviation/420f10013.htm

Environmental Protection Agency. (2011) Lead in air. Retrieved from http://epa.gov/airquality/lead/health.html

Harvey, A.D. (2006). Air records and war flying. Air Power History 53(3), 35-39.

Howden, S., Schneider, C., \& Grosser, Z. (1996). The analysis of soil, paint, and house dust for lead contamination. Atomic Spectroscopy 17(4), 171-175.

Illinois Department of Transportation. (2012). Airport inventory report. Retrieved from http://www.dot.state.il.us/aero/2012inventory.pdf

Institute of Medicine. (1998). The future of the public's health in the $21^{\text {st }}$ century. Washington, D.C.: National Academies Press.

Materials Analytical Services, Inc. (n.d.). Environmental lead sampling procedures. Retrieved from http://www.mastest.com/pdf/LeadSamplingMethod.pdf

Matsui, A. (2007). Case study: Switch to unleaded gas. PBS Newshour. Retrieved from http://www.pbs.org/newshour/indepth_coverage/science/alt_fuels/unleaded.html

Midgely, T. (1937). From the periodic table to production. Industrial and Engineering Chemistry 29(2), 41-44. 
Schaufelle, R. D. (2008). FAA aerospace forecast. Washington, DC. Retrieved from http://www.faa.gov/data_research/aviation/aerospace_forecasts/2009 $-2025 /$ media/2009\%20Forecast\%20Doc.pdf

Taylor, C., Park, K., Murphy, K., \& Mortvedt, T. (2010) Lead and halogen contamination from aviation fuel additives at Brackett Airfield. Retrieved from http://ea.pomona.edu/1151-2/student-research-funded-by-the-mellon-foundation/ 